\title{
ULTRA-HIGH-ENERGY COSMIC RAYS IN THE MAGELLANIC CLOUDS
}

\author{
R J. PROTHEROE \\ Department of Physics and Mathematical Physics \\ University of Adelaide \\ South Australia 5001 \\ Australia
}

ABSTRACT. Ultra-high-energy (UHE) $\gamma$-rays have been detected from several X-ray binaries. UHE $\gamma$-rays from sources in the LMC will interact in the microwave background producing electrons which emit synchrotron radiation in the $\mathrm{X}$-ray and $\gamma$-ray bands. This radiation might be observable.

\section{Introduction}

Several X-ray binaries have now been observed to be sources of UHE $\gamma$-rays (e.g. Nagle $e t$ al. 1988). UHE $\gamma$-rays will be attenuated by photon-photon pair production on traversing the microwave background; the electrons produced may inverse Compton scatter microwave photons to ultra-high energies leading to a cascade (see e.g. Protheroe 1986). The UHE $\boldsymbol{\gamma}$-rays produced in sources probably result from $\pi^{0}$ decay, with roughly equal numbers of $\pi^{+}, \pi^{-}$and $\pi^{0}$ being produced. The charged pions would decay via muons into electrons which, if they escape from the source, will also initiate cascades. During the cascade initiated by UHE $\gamma$-rays or electrons, synchrotron radiation is emitted in a continuous spectrum extending from $\mathrm{X}$-ray to $\gamma$-ray energies and would appear as a faint halo surrounding the UHE $\gamma$-ray source extending with diminishing brightness to several degrees from the source. The luminosity of the halo depends on the luminosity of the source in $\gamma$-rays in the energy region above $0.1 \mathrm{PeV}$ where photon-photon collisions occur.

\section{The cascade calculation and results}

Full details of the calculation are given by Protheroe $(1986,1990)$. The code is three-dimensional and treats fully photon-photon pair production and inverse Compton scattering of UHE electrons on the microwave background. The motion of electrons depends on the magnetic field configuration in the LMC. Studies of stellar polarization (Mathewson and Ford 1970) indicate the existence of a non-random component and this may be part of a "Panmagellanic" field (Schmidt 1970) possibly of fossil origin. Synchrotron emission at $1.4 \mathrm{GHz}$ extends beyond the stellar distributions of the LMC and SMC (Haynes et al. 1986). Based on radio continuum and polarization data there is evidence of turbulence scales in the magnetic structure of the LMC (Spicker and Feitzinger 1987). The field model used in the present work is that of Rand and Kulkarni (1989) for our own Galaxy in which the random component of the magnetic field 
dominates, being about $5 \mu \mathrm{G}$, and is constant over cells of size $\sim 55 \mathrm{pv}$. In the absence of a detailed model for the LMC field this model is applied to the LMC under one of two extreme assumptions: (i) the field is present only in the LMC; (ii) the field extends as far as our Galaxy. The true picture will probably be between these two.

I use as the prototype source, LMC X-4 for which I assume an $\mathrm{E}^{-2}$ UHE photon spectrum with $\mathrm{E} \mathrm{F}(>\mathrm{E}) \approx 48 \mathrm{eV} \mathrm{cm}^{-2} \mathrm{~s}^{-1}$ (Protheroe and Clay 1985). The intensities of photons in the halo around LMC X-4 are plotted in Figure 1 against angular distance from the source for three energy bands. The estimated background intensities are plotted as horizontal arrows (see Protheroe 1990 for references to data). At $100 \mathrm{MeV}$ the expected flux, due to interactions of low energy cosmic rays with interstellar gas in the LMC (Ginzburg and Ptuskin 1984), is comparable to that predicted here in the halo around LMC X-4 (indicated by "GP" in Figure 1). Observations at high angular resolution made with new generation $X$-ray and $\gamma$-ray telescopes under development may detect halos around UHE sources in the LMC and give an indication of UHE cosmic ray production.

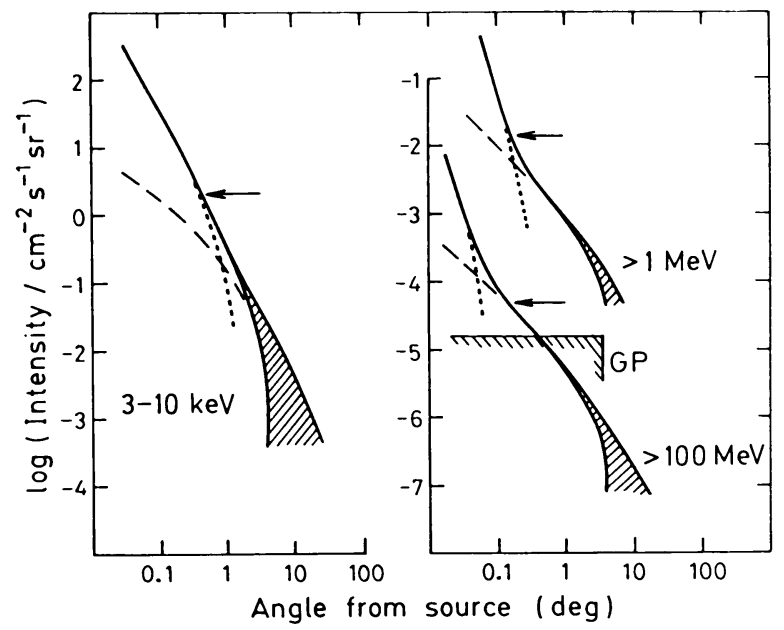

Figure 1. Halo around LMC X-4. Contributions of UHE electrons (dotted lines) and $\gamma$-rays (dashed lines) are indicated. Hatched areas indicate differences between models in which magnetic field is or is not confined to LMC (see text for details); arrows indicate estimated background intensities.

\section{References}

Ginzburg, V.W. and Ptuskin, V.S. (1984), J. Astrophys. Astr. 5, 99.

Haynes, R.F., Klein, V., Wielebinski, R. and Murray, J.D. (1986), Astron. Astrophys. 159, 22. Mathewson, D.S. and Ford, V.L. (1970), Ap. J. 160, L43.

Nagle, D.E. et al. (1988), Ann. Rev. Nucl. Part. Sci. 38, 609.

Protheroe, R.J. and Clay, R.W. (1985), Nature 315, 205.

Protheroe, R.J. (1986) Mon. Not. R. astr. Soc. 221, 769.

Protheroe, R.J. (1990) Mon. Not. R. astr. Soc. in press.

Rand, R.J. and Kulkarni, S.R. (1989) Ap. J. 343, 760.

Schmidt, Th. (1970), Astron. Astrophys. 6, 294.

Spicker, J. and Feitzinger, J.W. (1987), Interstellar Magnetic Fields ed. R. Beck and R. Grave (Springer-Verlag: Berlin), p.82. 